\title{
Hemodialysis Initiation in Oldest-Old Patients: A Case Series
}

\author{
Ryoichi Maenosono $^{a}$ Tomohisa Matsunaga ${ }^{a}$ Yuki Yoshikawa ${ }^{a}$

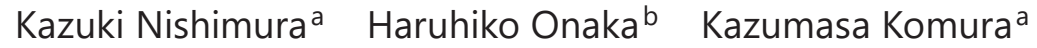 \\ Hirofumi Uehara ${ }^{a}$ Haruhito Azuma ${ }^{a}$ \\ aDepartment of Urology, Faculty of Medicine, Osaka Medical and Pharmaceutical University, \\ Osaka, Japan; bepartment of Cardiology, Takatsuki Red Cross Hospital, Osaka, Japan
}

\section{Keywords}

Oldest-old · Hemodialysis · Japan · End-stage renal disease

\begin{abstract}
With an increase in the number of older adults worldwide, the oldest-old population, defined as individuals over the age of 90 , is also growing. Japan is now facing the problem of a "superaged society" in which over $21 \%$ of the population is aged over 65 . The oldest-old constituted $1.8 \%$ (2.31 million) of the Japanese population in 2019. Such individuals have special healthcare needs. In cases of acute or chronic (or both) renal failure in the oldest-old, it becomes difficult to decide whether dialysis should be initiated. The issue is controversial, and there is some debate on whether dialysis should be avoided in elderly people because of their frailty or if it should be initiated to enable them to spend their remaining years with their families by improving their quality of life. Herein, we describe our experience in 4 cases of hemodialysis initiated in patients over the age of 90 . In our experience, dialysis enabled them to spend the rest of their lives with their families, which could not have been possible without it. Although further studies are needed, we concluded that oldest-old individuals in good general health could be eligible for and benefit from hemodialysis.
\end{abstract}

\section{Introduction}

An "elderly" or older person is defined as an individual over the age of 65 years. The elderly population is increasing worldwide. According to the World Health Organization, the proportion of the world's population over 60 years will nearly double from 12 to $22 \%$ between

\section{Karger!}


Table 1. Backgrounds of the 4 patients in whom hemodialysis was initiated after the age of 90 years

\begin{tabular}{lllll}
\hline & Case 1 & Case 2 & Case 3 & Case 4 \\
\hline Age, hemodialysis initiation & 91 & 92 & 90 & 94 \\
Age, vascular access & 91 & 92 & 89 & 94 \\
$\begin{array}{l}\text { Physical Examination, at initiation } \\
\text { Sex }\end{array}$ & Female & Male & Male & Male \\
$\quad$ Height, m & 1.46 & 1.62 & 1.57 & 1.60 \\
$\quad$ Body weight, kg & 42.4 & 76.0 & 58.1 & 61.3 \\
BMI & 19.9 & 29.1 & 23.6 & 23.9 \\
Primary disease & DMN & AKI & DMN & NS \\
Laboratory Examination, at initiation & & & & \\
$\quad$ Hemoglobin, g/dL & 9.9 & 9.5 & 9.6 & 10.2 \\
$\quad$ Serum albumin, g/dL & 2.3 & 3.7 & 3.4 & 4.1 \\
$\quad$ Brain natriuretic peptide, pg/mL & 453.8 & 907.2 & 189.6 & 262.9 \\
Emergency initiation? & Yes & Yes & No & No \\
CCI & 6 & 5 & 4 & 3 \\
SGA & C & C & A & B \\
GNRI, at initiation & 72.0 & 110.3 & 95.3 & 106.5 \\
GNRI, after 1 M & 75.7 & 83.0 & 94.3 & 95.6 \\
\hline
\end{tabular}

AKI, acute kidney injury; Alb, albumin; BMI, body mass index; BW, body weight; CCI, Charlson comorbidity index; DMN, diabetic nephropathy; GNRI, geriatric nutritional risk index; IBW, ideal body weight; SGA, subjective global assessment.

${ }^{*}$ GNRI was calculated using the formula: GNRI $=(1.489 \times \mathrm{Alb})+41.7 \times(\mathrm{BW} / \mathrm{IBW})[3]$.

2015 and 2050 [1]. Japan is now facing the problem of a "super-aged society" wherein over $21 \%$ of the population comprises individuals aged $>65$ years. Moreover, according to the Japanese Ministry of Internal Affairs and Communications, 28.4\% (35.88 million) of the Japanese population is aged over 60 years. In 2019, individuals over 90 years constituted $1.8 \%$ (2.31 million) of the Japanese population.

Generally, elderly individuals are frail, which is defined as multisystem impairment with increased vulnerability to stressors [2] due to age-related changes and are unsuitable candidates for high-risk operations, more so if they are aged over 90 years. However, whether individuals aged over 90 years should be treated with hemodialysis remains controversial. Herein, we narrate our experience with 4 cases of hemodialysis in patients aged over 90 years and then discuss whether hemodialysis initiation was suitable in these cases.

\section{Case Presentation}

We treated 4 chronic kidney disease (CKD) nonagenarians at our department between 2016 and 2020. Written informed consent was obtained from all patients. The patients' backgrounds and survival outcomes are summarized in Table 1 and Figure 1, respectively. Concerning the preservation period of renal failure, we referred to Ottawa Personal Decision Guides (OPDG) to confirm their willingness. We evaluated each patient's general condition with the Charlson comorbidity index (CCI), subjective global assessment (SGA), and Geriatric nutritional risk index (GNRI). 
Case Reports in Nephrology and Dialysis

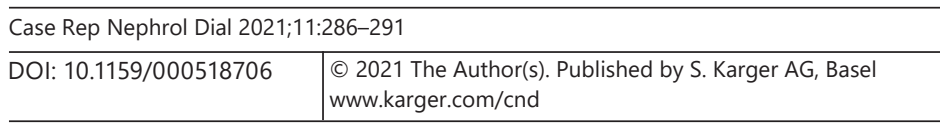

Maenosono et al.: Dialysis in the Nonagenarian
Fig. 1. Overall survival curve of our 4 oldest-old patients on hemodialysis.

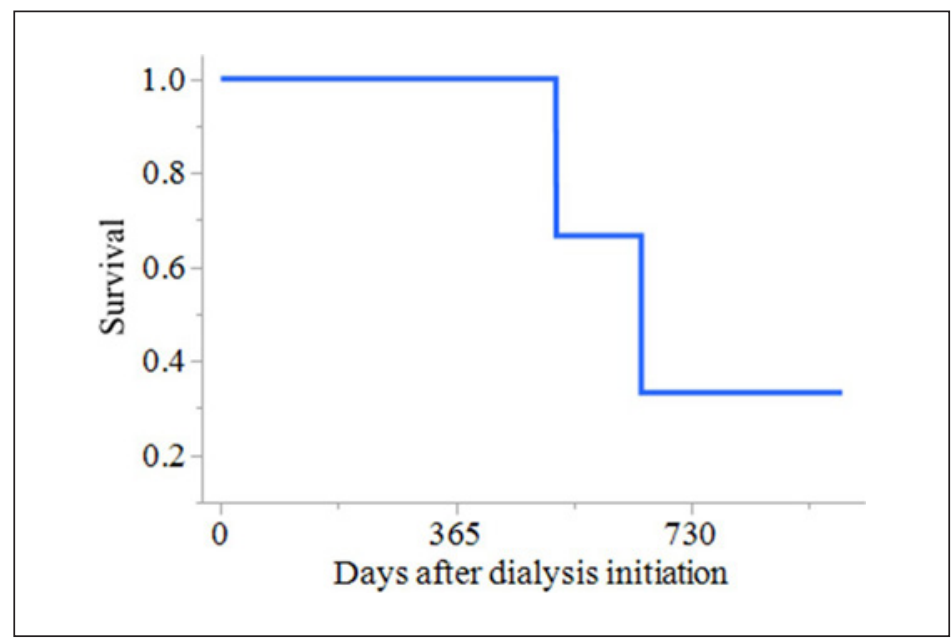

\section{Patient 1}

A 91-year-old woman with a 40-year history of diabetes mellitus gradually developed azotemia (blood urea nitrogen [BUN] $102 \mathrm{mg} / \mathrm{dL}$ ) and uremia requiring emergency hemodialysis. Although she was mostly in a wheelchair due to geriatric frailty, uremia also deprived her of her activity and appetite (CCI, 6; SGA, C; GNRI, 72.0). Prior to hemodialysis initiation, her attending physician presented the best supportive care without any dialysis as terminal care. Informed consent was obtained from this patient and her family after explaining the risks and benefits of hemodialysis. Her fatigability and appetite improved after hemodialysis; a GNRI of 87.5 at 1 year indicated improvement in her nutritional condition. After 1 year, she often experienced vascular access failure that caused inadequate dialysis; thus, her condition gradually deteriorated. She lived for 2 years.

\section{Patient 2}

A 92-year-old man with an abdominal aortic aneurysm suddenly developed a re-rupture of aneurysm after endovascular aortic repair. Although he had dementia and needed supportive care from his family (CCI, 5; SGA, C; GNRI, 110.3), there was no history of renal dysfunction (BUN $13 \mathrm{mg} / \mathrm{dL}$, eGFR $56 \mathrm{~mL} / \mathrm{min} / 1.73 \mathrm{~m}^{2}$ ). An emergency stent was placed in the renal artery, which soon caused acute kidney injury (BUN $46 \mathrm{mg} / \mathrm{dL}$ with anuria). The patient, however, could not express his real willingness under such conditions; he could not refer to OPDG to decide his treatment. After several discussions between his family and medical staff about risks and benefits, hemodialysis was initiated under shared decision-making. These emergent operations saved his life temporarily, but sudden changes caused physical debilitation and reduced his physical activity level. Although vascular access was unproblematic, he eventually underwent percutaneous endoscopic gastrostomy after hemodialysis initiation; GNRI was 74.5 at 6 months. To ensure optimal supportive care, he was moved to a hospice 6 months after the emergency hemodialysis initiation.

\section{Patient 3}

A 94-year-old man with hypertension and decreased renal function due to nephrosclerosis was referred to our hospital for follow-up. His condition and options had been thoroughly discussed with the patient and his family during the preservation period. A vascular access was placed in the left wrist prior to hemodialysis. Subsequently, he was transferred to our emergency department due to azotemia (BUN $108.8 \mathrm{mg} / \mathrm{dL}$ ) and severe uremia, which 
prompted hemodialysis after obtaining informed consent (CCI, 4; SGA, A; GNRI, 95.3). Even though he initially agreed to undergo dialysis, he became reluctant later on; fatigue and restriction while on dialysis caused psychological distress. Hemodialysis treatment prolonged his life for one and a half years without any cardiovascular problems, while hemodialysis was discontinued in deference to his wishes. Thereafter, he was admitted to a hospice to spend his remaining days under good supportive care.

\section{Patient 4}

A 90-year-old man with diabetes mellitus and drug-induced nephropathy exhibited a willingness to undergo hemodialysis through the creation of vascular access during the previous year, owing to a supportive family. He had a medical history of angina and laryngeal cancer for which he had received treatment. He had also undergone endovascular aortic repair for a common iliac artery aneurysm. This case was thoroughly discussed among the patient, his family, his attending physician, and other medical staff during the preservation period of renal failure. Hemodialysis was initiated when the BUN level reached $96.1 \mathrm{mg} / \mathrm{dL}$, and he started exhibiting signs of uremia (CCI, 3; SGA, A; GNRI, 106.5). The patient is still alive and has been undergoing hemodialysis for two and a half years (present age, 93 years). He has good adherence to our medication, and eventually can undergo a well-managed dialysis regimen; he spends quality time with his family, who provide the best supportive care and are satisfied with his treatment. Hemodialysis initiation was suitable for this patient.

\section{Discussion}

In this report, we described 4 patients aged over 90 years with different backgrounds who underwent hemodialysis. As the aging population increases, the number of oldest-old patients with end-stage renal disease (ESRD) will also increase. According to the Japanese Society for Dialysis Therapy, there were only 2 patients $(0.005 \%$ of all patients) aged over 90 years on hemodialysis in 1982, increasing to $2.3 \%$ in 2018 [4]. Aside from Japan, several other countries are now facing the problem of an "aged society" [5]. Thus, it can be predicted that more oldest-old people with ESRD will undergo hemodialysis treatment.

Developments in medicine have made it possible to provide improved care to the oldestold, thereby increasing their life expectancy, which was not possible earlier. They have also slowed the progression of chronic disease toward disability. Some reports have expressed criticism [6] regarding the initiation of hemodialysis in the hyper-aged society, citing the increased burden on healthcare, issues pertaining to cost-effectiveness [7], or ethics [8] as reasons. Factors such as malnutrition [3], low activities of daily living (ADL) [9], emergent initiation [10], or comorbidities [11] could contribute to vulnerable outcomes; such factors increase with age. In this regard, patients 3 and 4, who exhibited good ADL and nutritional status (SGA and GNRI) and no emergent initiation, revealed better outcomes. Patient 4, especially, is currently in good health and undergoing regular dialysis with the support of his family, suggesting that age should not be a barrier to hemodialysis initiation [12]. In contrast, patient 1 might have been too frail to undergo hemodialysis due to poor functional and nutritional status and the presence of comorbidities (high CCI). Such patients are unsuitable for hemodialysis in our experience. The most challenging case was that of patient 2 . Such cases could generate controversy regarding the decision to initiate hemodialysis [8]. In contrast, patient 1 had poor ADL scores; though the BUN levels improved, the patient gradually became weak. All physicians should ensure that the rights of elderly people with ESRD regarding the potential benefits and risks of hemodialysis are respected through a shared decision-making

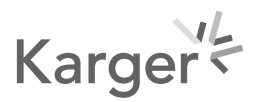


process at the early treatment stage and the end of life [13], and their functional and nutritional status and comorbidities are considered prior to arriving at a decision [14]. There were no troubles in 3 cases while discussing with the patients' families and medical staff for initiation dialysis. Almost all cases were referred to OPDG to confirm their willingness. However, it was difficult to decide to start hemodialysis for the case 2 patient. Although initiation of hemodialysis is medically considered by nephrologists when one or more CKD symptoms are present, an aggressive trial of nondialytic management of advanced symptoms might be warranted depending on the patient's preferences and circumstances. Thus, patients and their families must be informed of conservative management options, such as supportive care and hospice care [15].

This study has some major limitations, such as a small study sample, unclear criteria for hemodialysis initiation, and the lack of objective data regarding sarcopenia or frailty. Further, more clinical data are required to determine the validity of hemodialysis initiation in oldestold people and decide whether hemodialysis or supportive care is better for elderly patients with ESRD. This issue is difficult and remains controversial [15], though it may be beneficial to initiate hemodialysis in patients with good general health.

Appropriate treatment, along with strong family support, can greatly improve a patient's health and quality of life. Studies on hemodialysis initiation and supportive care have revealed heterogeneous results due to a paucity of survival data for supportive care patients [15]. Further studies are warranted to ensure whether elderly CKD patients should undergo hemodialysis and determine factors considered for initiation.

\section{Acknowledgments}

We would like to express our deepest gratitude to Dr. Maki Kagitani, Dr. Hajime Hirano, and Dr. Hayahito Nomi, Osaka Medical and Pharmaceutical University Hospital Blood Purification Center, for their discussions and insight. We also appreciate Yukiko Kusunoki and the staff working at the Blood Purification Center for their highly skilled management of the patients. Finally, we thank Editage (www.editage.com) for English language editing.

\section{Statement of Ethics}

This case report did not require an approval from the Osaka Medical and Pharmaceutical University Ethics Committee as the study did not include any invasive investigation or clinical trial. The case study conformed to the guidelines of the Declaration of Helsinki. Written informed consent was obtained from the patients for publication of this case series.

\section{Conflict of Interest Statement}

The authors have no conflicts of interest to declare.

\section{Funding Sources}

This research received no specific grant from any funding agency in the public, commercial, or not-for-profit sectors.

\section{Karger'}




\section{Case Reports in Nephrology and Dialysis}

\begin{tabular}{l|l}
\hline Case Rep Nephrol Dial 2021;11:286-291 \\
\hline DOI: 10.1159/000518706 & $\begin{array}{l}\text { @ 2021 The Author(s). Published by S. Karger AG, Basel } \\
\text { www.karger.com/cnd }\end{array}$ \\
\hline
\end{tabular}

Maenosono et al.: Dialysis in the Nonagenarian

\section{Author Contributions}

R.M. performed analyzed data and wrote the manuscript. T.M., Y.Y., S.T., and H.O. collected and analyzed data. H.U. and K.K. helped with the preparation of the manuscript. H.A. supervised the project, wrote, and edited the manuscript.

\section{Data Availability Statement}

All data generated or analyzed during this study are included in this article. Further enquiries can be directed to the corresponding author.

\section{References}

1 World Health Organization. Ageing and health [Internet]. 2018 [cited 2018 Feb 5]. Available from: https:// www.who.int/news-room/fact-sheets/detail/ageing-and-health.

2 Clegg A, Young J, Iliffe S, Rikkert MO, Rockwood K. Frailty in elderly people. Lancet. 2013 Mar;381(9868): 752-62.

3 Bouillanne O, Morineau G, Dupont C, Coulombel I, Vincent JP, Nicolis I, et al. Geriatric nutritional risk index: a new index for evaluating at-risk elderly medical patients. Am J Clin Nutr. 2005 Oct;82(4):777-83.

4 Nitta K, Goto S, Masakane I, Hanafusa N, Taniguchi M, Hasegawa T, et al. Annual dialysis data report for 2018, JSDT renal data registry: survey methods, facility data, incidence, prevalence, and mortality. Ren Replace Ther. 2020 Dec;6(1):1-8.

5 Canaud B, Tong L, Tentori F, Akiba T, Karaboyas A, Gillespie B, et al. Clinical practices and outcomes in elderly hemodialysis patients: results from the dialysis outcomes and practice patterns study (DOPPS). Clin J Am Soc Nephrol. 2011 Jul;6(7):1651-62.

6 Thorsteinsdottir B, Swetz KM, Albright RC. The ethics of chronic dialysis for the older patient: time to reevaluate the norms. Clin J Am Soc Nephrol. 2015 Nov;10(11):2094-9.

7 Hall RK, Myers ER, Rosas SE, O'Hare AM, Colón-Emeric CS. Choice of hemodialysis access in older adults: a cost-effectiveness analysis. Clin J Am Soc Nephrol. 2017 Jun;12(6):947-54.

8 Akbar S, Moss AH. The ethics of offering dialysis for AKI to the older patient: time to re-evaluate? Clin J Am Soc Nephrol. 2014 Sep;9(9):1652-6.

9 Bossola M, Di Stasio E, Antocicco M, Pepe G, Tazza L, Zuccalà G, et al. Functional impairment is associated with an increased risk of mortality in patients on chronic hemodialysis. BMC Nephrol. 2016 Dec;17(1):72.

10 Roy D, Chowdhury AR, Pande S, Kam JW. Evaluation of unplanned dialysis as a predictor of mortality in elderly dialysis patients: a retrospective data analysis. BMC Nephrol. 2017 Dec;18(1):364.

11 Murtagh FE, Marsh JE, Donohoe P, Ekbal NJ, Sheerin NS, Harris FE. Dialysis or not? A comparative survival study of patients over 75 years with chronic kidney disease stage 5. Nephrol Dial Transplant. 2007 Jul;22(7): 1955-62.

12 Shrestha A, Burns A. 103-Year-old on dialysis: was it worth it? BMJ Case Rep. 2017 Jan;2017:bcr2016216493.

13 Santos J, Oliveira P, Malheiro J, Campos A, Correia S, Cabrita A, et al. Predicting 6-month mortality in incident elderly dialysis patients: a simple prognostic score. Kidney Blood Press Res. 2020 Jan;45(1):38-50.

14 Chan CT, Blankestijn PJ, Dember LM, Gallieni M, Harris DCH, Lok CE, et al. Dialysis initiation, modality choice, access, and prescription: conclusions from a kidney disease: improving global outcomes (KDIGO) controversies conference. Kidney Int. 2019 Jul;96(1):37-47.

15 Foote C, Kotwal S, Gallagher M, Cass A, Brown M, Jardine M. Survival outcomes of supportive care versus dialysis therapies for elderly patients with end-stage kidney disease: a systematic review and meta-analysis. Nephrology. 2016 Mar;21(3):241-53. 